DOI: $10.12731 / 2306-1561-2013-4-35$

\title{
CONCEPTUAL FOUNDATIONS OF INTELLIGENT REMOTE MONITORING AND CONTROL FACILITIES SPECIAL PURPOSE
}

\section{Nikonov V.V.}

\section{Abstract}

In article proposes the concept of intelligent remote monitoring and control of special purpose by creating appropriate methods, algorithms and methodologies, as well as the Information Technology industry. The application of artificial intelligence methods to the problem of identification of harmful substances and the establishment of their concentration on the basis of data obtained from laser systems for remote sensing. Established expert information system on the basis of non-monotonic inference to identify harmful substances. To control the consistency of the facts in the EIS proposed to use a support system for reasoning. We propose a neural network method to establish the concentration of harmful substances on the basis of the IR absorption spectra of compounds emissions. The proposed concept of intelligent remote monitoring and control of special-purpose and complex information technology can be used to determine the presence of hazardous substances and their concentration in the air, using data obtained from the IR laser systems sensing systems for environmental monitoring and control of special purpose ; to monitor the presence of harmful substances highways and other cities.

Keywords: concept, modified reasoning formal-conceptual analysis, expert information system, approximation, automated system, environmental monitoring, hazardous substances, neural networks, artificial intelligence.

\section{УДК 004.8}

\section{КОНЦЕПТУАЛЬНЫЕ ОСНОВЫ ИНТЕЛЛЕКТУАЛЬНОГО ДИСТАНЦИОННОГО МОНИТОРИНГА И КОНТРОЛЯ ОБЪЕКТОВ СПЕЦИАЛЬНОГО НАЗНАЧЕНИЯ}

\section{Никонов В.В.}

\section{Аннотация}

В статье предлагается конщепция интеллектуального дистанциионного мониторинга и контроля объектов специального назначения путем создания соответствующих методов, алгоритмов и методик, а также информачионнотехнического комплекса. Рассматривается применение методов искусственного интеллекта к задаче идентификащии вредньх веществ и установлении их концентрации на основе данных, полученных от систем дистанционного лазерного 
зондирования. Создана экспертно-информационная система на основе немонотонного логического вывода для идентификации вредных вещуеств. Для управления непротиворечивостью фактов в ЭИС предложено использовать систему поддержки рассуждений. Предложен нейросетевой метод установления концентрации вредных вещуеств на основе ИК-спектров поглощуения соединений газов. Предлагаемая концепция интеллектуального дистанционного мониторинга и контроля объектов специального назначения в виде информационно-технического комплекса может быть использована для определения наличия вредных веществ и их концентрации в воздушной среде с использованием данных, полученных от систем лазерного ИК зондирования; в системах экологического мониторинга и контроля объектов специального назначения; для мониторинга наличия вредных веществ автомобильньхх трасс городов и др.

Ключевые слова: концепция, модифицированные рассуждения, формальныйконцептуальный анализ, экспертно-информационная система, аппроксимаџия, автоматизированная система, экологический мониторинг, вредные вещества, нейронные сети, искусственный интеллект.

\section{Введение}

Постоянное получение оперативной информации о наличии и содержании отравляющих веществ в атмосфере, продуктов их детоксикации и общепромышленных загрязнителей в контролируемых зонах, о возможном их поступлении в окружающую среду, а также о динамике изменения уровня их концентраций необходимо для контроля безопасности, для оперативного принятия решения по предотвращению поступления отравляющих веществ, продуктов их детоксикации и общепромышленных загрязнителей в окружающую природную среду при нарушениях технологического процесса, а также для оповещения персонала и населения в случае возникновения аварийных ситуаций.

Мониторинг поступления в окружающую среду различных концентраций аэрозольных загрязнений необходим для оперативного принятия решения по предотвращению поступления отравляющих веществ в окружающую природную среду при нарушениях технологического процесса, а также для оповещения персонала и населения в случае возникновения аварийных ситуаций.

В настоящее время в России широко развертываются работы по изучению процессов, происходящих в окружающей среде, в том числе и под влиянием антропогенных факторов. Федеральный закон «Об охране окружающей среды» № 7-Ф3 от 10.01.2002 г., закон г. Москвы «Об экологическом мониторинге в г. Москве» № 65 от 20.10.2004 г. определяют необходимость разработки методов и средств контроля процессов, происходящих в окружающей среде.

Исследования по охране окружающей среды ведутся в различных областях науки и техники. Результаты этих исследований публикуются в разных изданиях. Число организаций занимающихся проблемами экологического мониторинга также велико. 
Об этом свидетельствует формируемая программа «экологической безопасности России». Однако практическое использование результатов этих исследований для решения экологических проблем сдерживается недостаточным развитием теории и практики построения экологических информационных систем и систем интеллектуального экологического мониторинга, представляющих собой один из новых видов автоматизированных систем и предназначенных для сбора и анализа разнородной информации о состоянии окружающей среды $[1,18,19]$.

\section{Концепция интеллектуальной поддержки принятия решений}

При исследованиях состояния атмосферы и ее загрязненности особое место занимают методы бесконтактного мониторинга, обеспечивающие возможность получения данных с высокой оперативностью и в значительных пространственных масштабах, позволяющие предоставить количественную информацию о загрязнениях воздуха и их природе. Таким требованиям удовлетворяют лидарные методы дистанционного зондирования, которые используют рассеяние и поглощение лазерного излучения атмосферными компонентами.

Лидарные системы инфракрасного дистанционного зондирования могут быть использованы для мониторинга окружающей среды, в системах специального экологического мониторинга объектов по уничтожению химического оружия и в системах промышленного экологического мониторинга радиоизлучений и др.

При дистанционном лидарном зондировании окружающей среды молекулы вредных веществ (газов и аэрозоли) являются причиной ослабления проходящего через нее лазерного излучения. Часть излучения рассеивается в обратном направлении (в сторону приемника лидара) на аэрозольных частицах, либо отражается от объектов или специально установленных экранов. Это излучение собирается и направляется на преобразователь, который преобразует его в электрический сигнал, пропорциональный интенсивности принятого оптического излучения.

Эффективным методом исследования воздушной среды атмосферы является метод лазерного инфракрасного (ИК) зондирования. Данный метод позволяет на основе принципов поглощения и рассеивания электромагнитного излучения получать данные о наличии и концентрации вредных веществ, что позволяет выявлять причины, механизмы, тенденции развития процессов, происходящих в атмосфере. Изучением обработки информации, полученной от систем лазерного зондирования воздушной среды занимаются такие отечественные ученые как: Агишев Р.P. [2], В.Е. Зуев, В.С. [3] Портасов, И.Д. Миценко, Г.Г. Ванеев, Е.С. Селезнева и др. Существующие в настоящее время методы и модели предназначены для изучения различных газов, температуры, давления, скорости, а также других параметров атмосферы. Эти методы трудоемки и требуют высокой квалификации персонала. Экспертные процедуры, в данном случае, строятся на определении наличия только одного вредного вещества. Поэтому возникает необходимость в разработке концепции, методов, моделей и алгоритмов для анализа данных, полученных от лазерных систем дистанционного зондирования воздушного 
бассейна атмосферы, что позволит определять одновременно несколько вредных веществ и их концентрацию в режиме реального времени без участия эксперта.

Концепция интеллектуального дистанционного экологического мониторинга и контроля объектов специального назначения основана на использовании современных методов искусственного интеллекта и состоит из трех основных этапов (рисунок 1): создание процедур бора информации с системы дистанционного лазерного зондирования, идентификация вредных веществ и определение концентрации вредных веществ $[5-7,18]$.

Дистанционный контроль можно определить как качественное или количественное измерение химического или физического параметра окружающего пространства, когда контролирующий прибор и объем, параметр которого исследуется, пространственно разделены [2]. Приборы дистанционного контроля могут обеспечить ту же точность, что и приборы для прямых измерений, кроме того, они имеют ряд уникальных преимуществ, а именно: допускают ряд специфических приложений, осуществление которых было бы затруднительно, если вообще возможно, при использовании стандартных приборов.

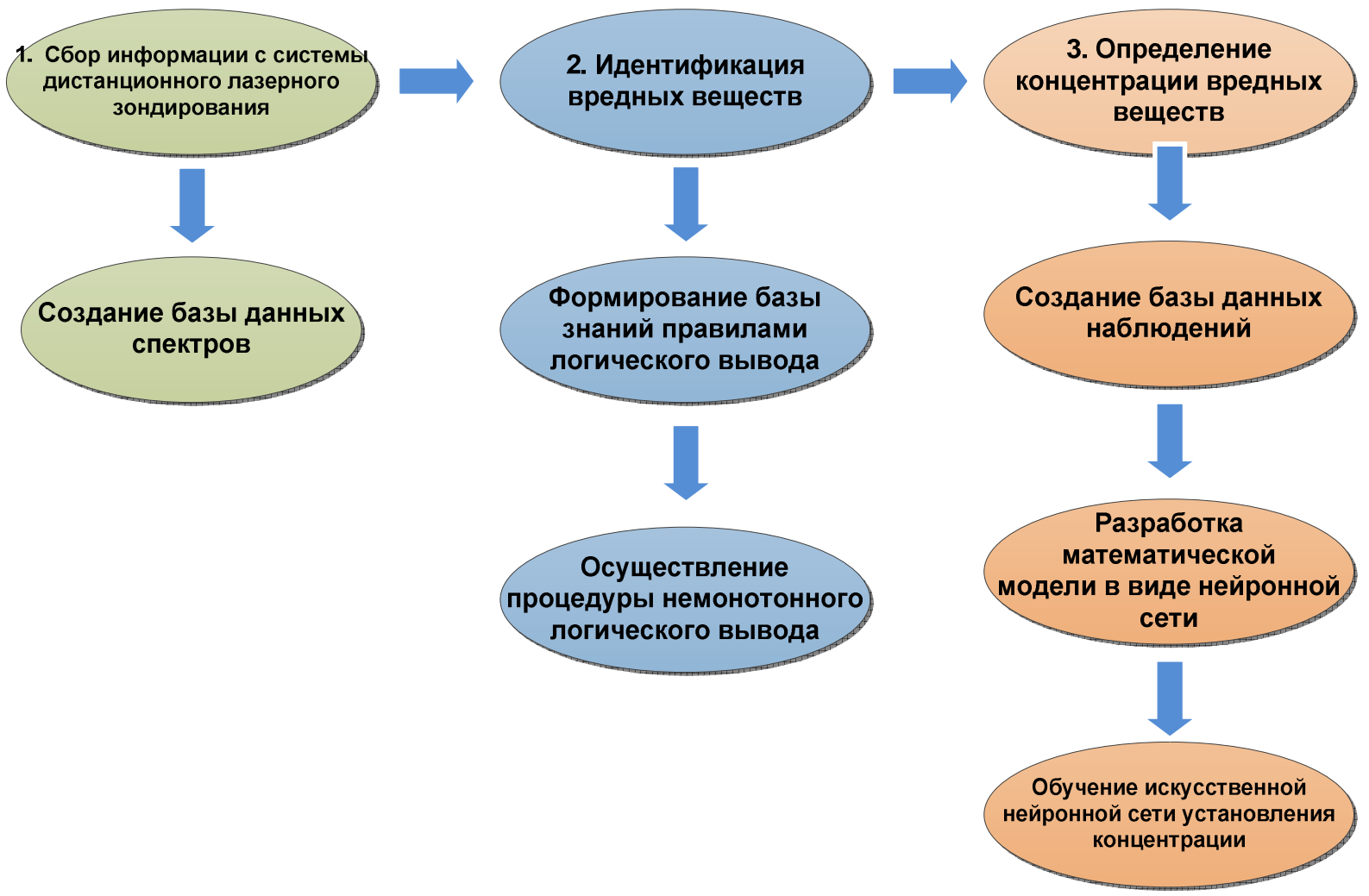

\section{Рисунок 1 - Концепция интеллектуального дистанционного экологического мониторинга и контроля объектов специального назначения}

\section{Сбор информации с системы дистанционного лазерного зондирования}

Дистанционные методы обеспечивают проведение измерений практически в любых пространственных масштабах, дают возможность проведения интегральных оценок (например, оценки общего содержания газов, либо оценки общей дозы 
загрязнения окружающей среды), позволяют проводить исследования обширных территорий, воздушных и водных бассейнов со спутников, самолетов и автомобилей.

Дистанционные методы не вносят в изучаемую среду возмущений, которые могут возникнуть при взятии проб и способны повлиять на результаты исследований. Кроме того, осуществление измерений с помощью зондов порой технически невозможно и экономически неэффективно.

Применение в области экологического контроля методов ИК спектроскопии на основе дистанционного зондирования в сочетании с уникальными свойствами лазерного излучения привело к созданию мощных систем мониторинга, зарекомендовавших себя с наилучшей стороны.

Использование свойств лазерного излучения, таких как, высокая спектральная плотность энергии, монохрамотичность, возможность плавной перестройки длины волны излучения, позволило использовать данные технологии при осуществлении дистанционного мониторинга воздушной среды, обладающие высокой чувствительностью и высоким спектральным разрешением [3].

Все лазерные газоанализаторы построены по схеме прямого детектирования, в которой детектором поглощенной мощности является сам образец.

Лазерный метод выгодно отличается от других методов следующими особенностями [5]:

- высокое спектральное разрешение, определяющееся шириной лазерного излучения;

- высокая чувствительность по показателю поглощения;

- отсутствие сигнала, если спектральная линия излучения лазерного источника находиться вне линии поглощения газа;

- отношение сигнал/шум увеличивается пропорционально мощности источника

- принципиальное ограничение на мощность налагается лишь явлением насыщения поглощения на исследуемой лини [2, 3];

- сигнал пропорционален показателю поглощения газа, что существенно упрощает обработку данных измерения;

- измеряемая величина (амплитуда колебания давления) является интенсивным параметром образца, т.е. не зависит от его размеров;

- шумы приемников из-за высокой спектральной плотности мощности лазерных источников, как правило, не имеют значения. Стабилизация интенсивности лазерного излучения (или нормировка на опорный сигнал) еще более увеличивает чувствительность аппаратуры;

- область, используемых в газоанализаторах лазерных источников, охватывает спектральный диапазон ИК-области.

- возможность быстрой перестройки длины волны излучения, что позволяет проводить оперативный количественный анализ многокомпонентных газовых смесей. 
Трудность в получении спектра поглощения инфракрасного излучения с системы дистанционного лазерного зондирования и автоматизация данного процесса заключается в формализации исходных данных, представленных спектром поглощения, типичный вид которого представлен на рисунке 2. В процессе спектрального анализа получают аналитический сигнал, который в регистрируемом спектре изменяет свое спектральное расположение в зависимости от связанных с ней волновых параметров: частоты (f), волнового числа (k), длина волны ( $\lambda)$ [3].

Очевидные преимущества лазерного дистанционного метода зондирования воздушного бассейна атмосферы в сочетании с использованием достаточно мощных непрерывных перестраиваемых по частоте лазеров делают его особенно привлекательным для решения задач, требующих измерения слабого поглощения излучения молекулярными газами. В первую очередь это касается задач газового анализа при малых и сверхмалых концентрациях молекул в среде.

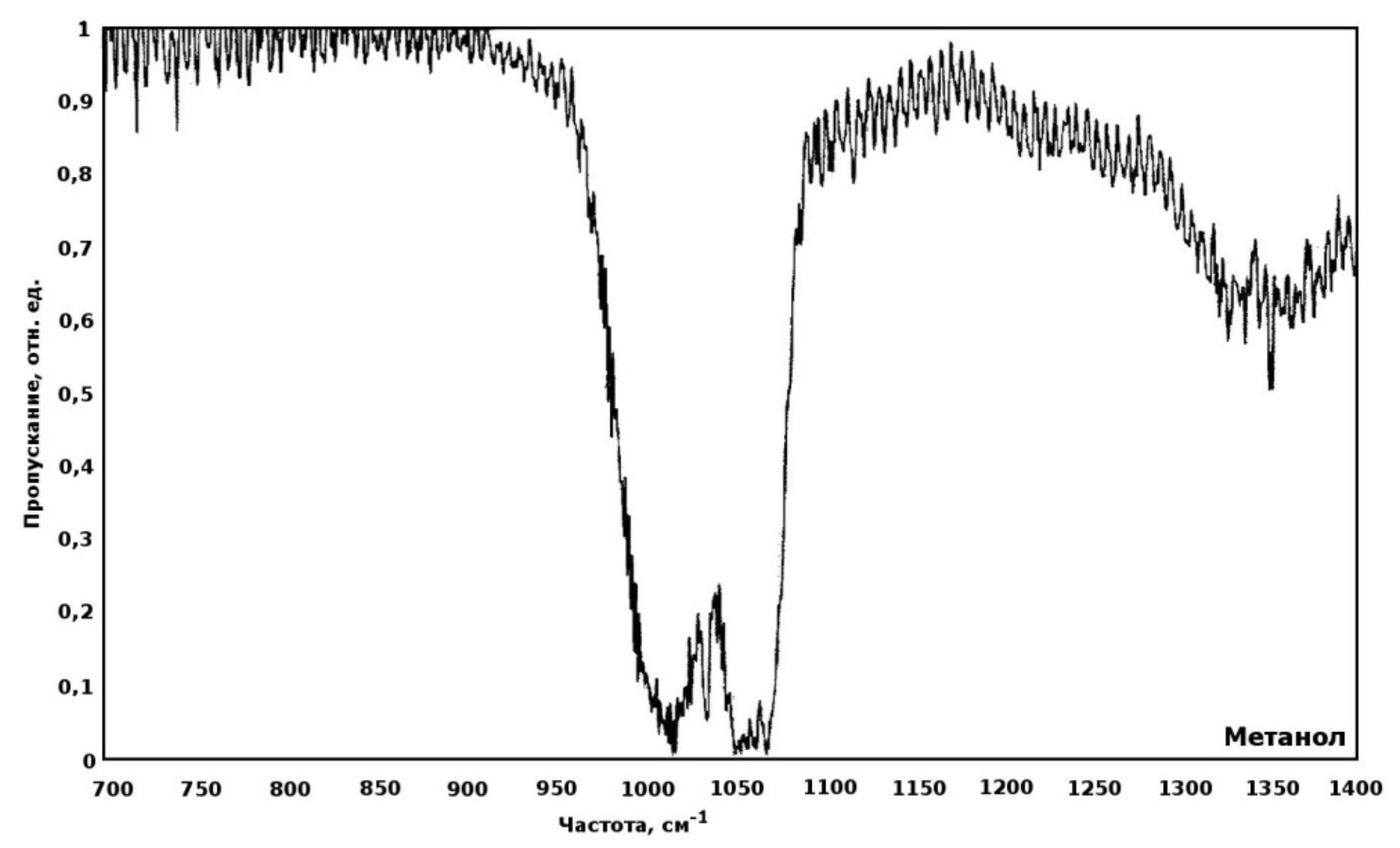

Рисунок 2 - Пример ИК спектра поглощения метанолом

Таким образом, перспективным на данный момент является использование в задаче определения количественного и качественного анализа возможностей искусственного интеллекта, главным преимуществом которого является обработка результатов измерений в условиях неопределенности, неполноты и большой размерности данных.

\section{Идентификация вредных веществ}

Следующим этапом является идентификация вредных веществ в воздушной среде. Для идентификации вредных веществ по данным лазерного ИК зондирования 
предлагается модель экспертно-информационной системы (ЭИС) на основе логического формализма модифицируемых рассуждений.

В рамках создания правил логического вывода, необходимых для принятия решений о наличии вредных веществ в окружающей среде разработан алгоритм создания правил на основе использования формального концептуального анализа (рисунок 3) [8-10, 17].

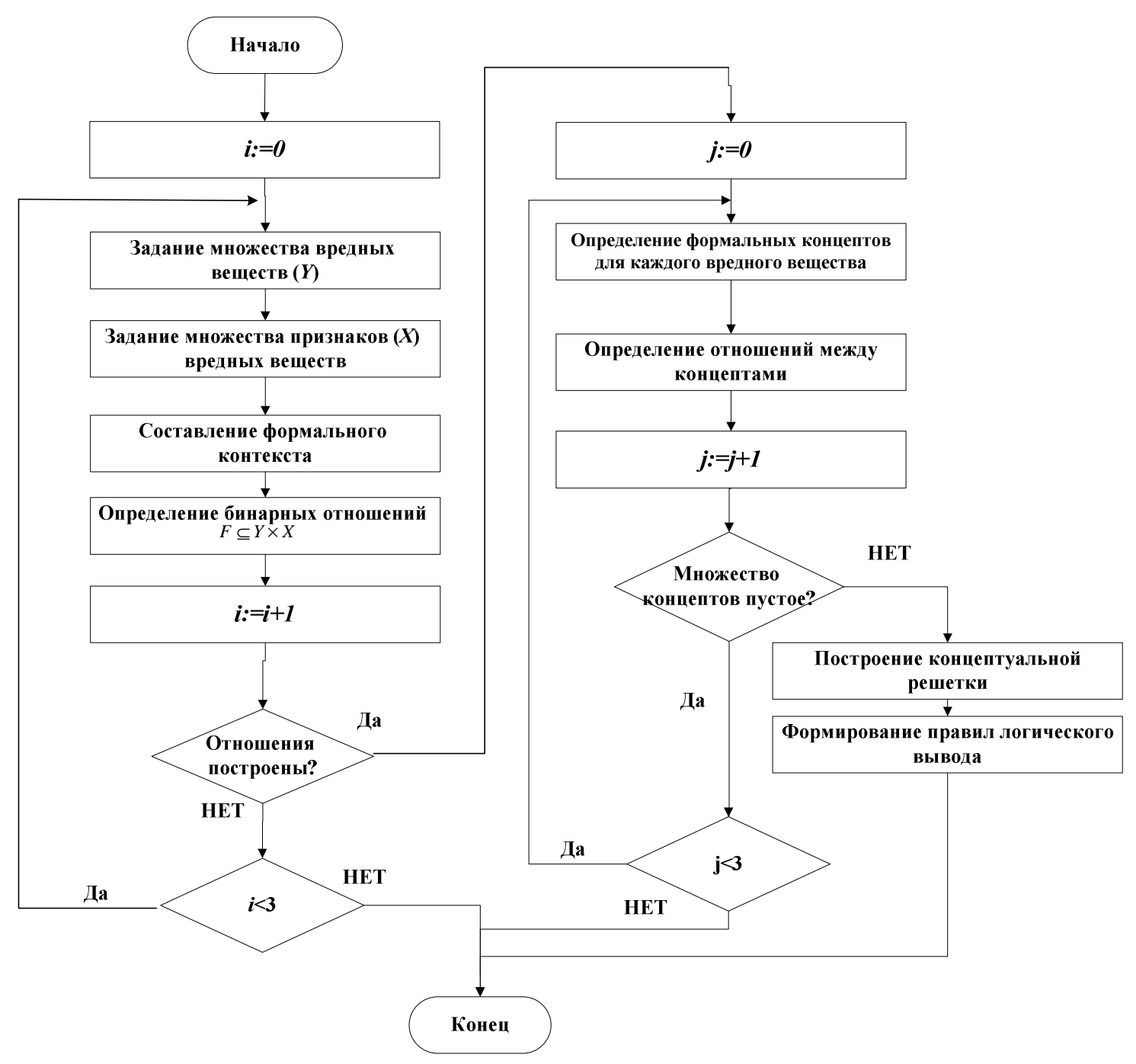

\section{Рисунок 3 - Блок-схема алгоритма создания правил логического вывода для определения вредных веществ}

Модель формального концептуального анализа базируется на теории решеток Бирхгофа, что позволяет алгоритмизировать данную модель [9-10].

Формальный контекст - это тройка (Y, X, F), которая состоит из множества объектов (вредных веществ в воздухе атмосферы) Y, множества атрибутов (признаков вредного вещества) $\mathrm{X}$ и бинарных отношений $F \subseteq Y \times X$ между объектами и атрибутами. 
На прямом произведении $\mathrm{Y} \times \mathrm{X}$ двух множеств существует частичный порядок, если $\left(y_{1}, x_{1}\right) \leq\left(y_{2}, x_{2}\right)$ тогда и только тогда, когда $y_{1} \leq y_{2}$ в Ү и $x_{1} \leq x_{2}$ в Х.

Решеткой называется множество L, в котором любые два его элемента имеют точную верхнюю грань, т.е. «объединение» $x \vee y$, и точную нижнюю грань, или «пересечение» $x \wedge y$.

Как правило, контекст имеет вид таблицы, в которой строки представляют собой объекты, а столбцы - атрибуты.

Разработанный алгоритм используется для разработки правил логического вывода идентификации вредных веществ в воздушной среде по спектру поглощения инфракрасного излучения.

На основе этого алгоритма строится контекст для формирования правил логического вывода, пример которого показан в таблице 1.

Таблица 1 - Пример контекста формирования логических правил.

\begin{tabular}{|c|c|c|c|c|c|c|c|c|c|}
\hline Атрибуты & & 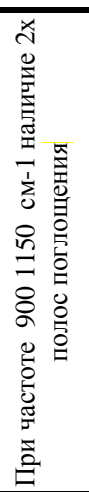 & 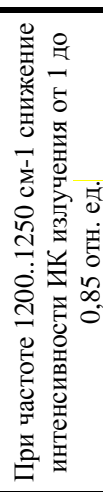 & 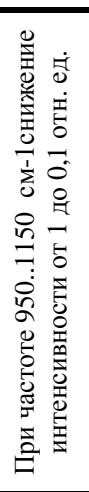 & 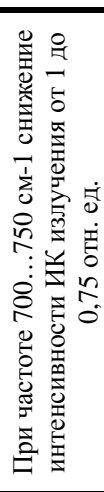 & 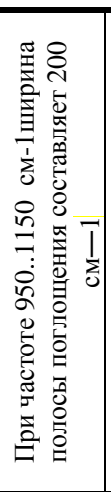 & 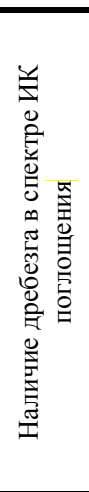 & 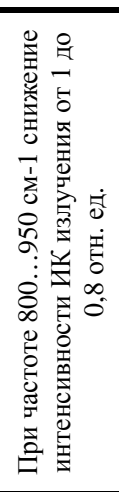 & 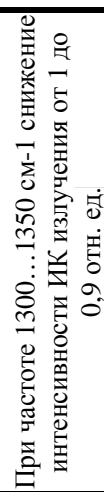 \\
\hline Метанол & $\mathrm{M}$ & & + & & & + & + & + & \\
\hline Этанол & Э & & + & + & + & + & + & + & + \\
\hline Аммиак & A & + & & + & & & + & + & + \\
\hline Диметилсульфоксид & Д & + & & + & & & & + & \\
\hline
\end{tabular}

На основе контекста и алгоритма разрабатывается формальная концептуальная решетка, для создания правил логического вывода. Пример формальной концептуальной решетки показан на рисунке 4. Данная решетка позволяет автоматизировать процесс построения правил логического вывода.

Например, бинарное отношение $(Э, 4)$ означает, что в случае, если в с воздушной среде присутствует этанол (объект) на спектре ИК поглощения для волновых чисел (при частоте) $700 \ldots 750 \mathrm{cm-1}$ наблюдается скачок значения уровня поглощения от $100 \%$ до $75 \%$ (атрибут). Таким образом, $(g, m) \in F$ означает, что «объект g обладает свойством m».

Под концептом (А,N) понимается пара объекта $A \subseteq Y$ и атрибута $N \subseteq X$, которые удовлетворяют определенным условиям. Чтобы определить необходимость и достаточность условий для формального концепта, введены два оператора, допустив $A \subseteq Y$ : 


$$
A^{\prime}=\{m \in X, \forall g \in A:(g, m) \in F\},
$$

где $\mathrm{m}$-атрибут конкретного объекта g, и соответственно для $N \subseteq X$ :

$$
N^{\prime}=\{g \in Y, \forall m \in N:(g, m) \in F\} \text {, }
$$

где $\mathrm{g}$-конкретный объект, обладающий атрибутом m.

Приведенные формулы (1) и (2) означают, что множество $A^{\prime}$ содержит все атрибуты, которые являются общими для всех объектов А, а множество $N^{\prime}$ есть множество всех объектов, которые обладают всеми свойствами множества $\mathrm{N}$.

Пара (A, N) есть формальный концепт, если выполняется условие:

$$
A^{\prime}=N_{\text {и }} A=N^{\prime} \text {. }
$$

Данное свойство означает следующее: все объекты концепта содержат все его атрибуты. Из определения формального концепта следует, что для всех $A \subseteq Y$ пара $\left(A^{\prime \prime}, A^{\prime}\right)$ есть формальный концепт и для всех $N \subseteq X$ пара $\left(N^{\prime}, N^{\prime \prime}\right)_{\text {тоже }}$ формальный концепт.

Для формальных концептов природа отношения подконцепт/надконцепт определена следующим образом:

$$
\left(A_{1}, N_{1}\right) \leq\left(A_{2}, N_{2}\right) \Leftrightarrow A_{1} \subseteq A_{2}, N_{1} \subseteq N_{2} .
$$

Это отношение выявляет дуализм между атрибутами и объектами концептов. Концепт $C_{1}=\left(A_{1}, N_{1}\right)$ является подконцептом концепта $C_{2}=\left(A_{2}, N_{2}\right)$, если множество его объектов являются подмножеством объектов $C_{2}$. Таким образом, множество всех формальных концептов образуют концептуальную решетку.

Решетка является моделью, по которой можно проследить все свойства, которыми обладает то или иное определяемое вредное вещество - это множество всех свойств, лежащих выше узла. Каждый узел решетки соответствует концепту. Графическим представлением концептуальной решетки является линейная диаграмма. Она позволяет исследовать и интерпретировать отношения между концептами, объектами и атрибутами, является эквивалентным представлением контекста. Она содержит точно такую же информацию, что и таблица отношений, в которой каждому узлу соответствует концепт из данного контекста.

На диаграмме каждый объект обладает свойствами, приписанными узлу, и свойствами узлов, с которыми этот узел связан дугами снизу вверх.

Построим концептуальную решетку для формирования логических правил для идентификации вредных веществ. В таблице 1 задан формальный контекст $\mathrm{K}=(\mathrm{Y}, \mathrm{X}, \mathrm{F})$, где $\mathrm{Y}$ - множество вредных веществ (для примера рассмотрено 4 вещества), X - их свойства, F - бинарное отношение между веществами и свойствами.

При построении решетки совпадающие столбцы таблицы (если такие есть) можно интерпретировать как наличие одного или/и другого признака. Поэтому при 
необходимости на линейной диаграмме второй признак заключается в скобки. Эта ситуация также может свидетельствовать о линейной зависимости между столбцами.

На основе контекста, представленного таблицей 1, строится формальная концептуальная решетка, для создания правил логического вывода (рисунок 4).

Граф состоит из узлов, которые представляют собой концепты, и ребра, соединяющих эти узлы. Два узла $C_{1}$ и $C_{2}$ соединены тогда и только тогда, когда $C_{1} \leq C_{2}$ и нет такого концепта $C_{3}$, что $C_{1} \leq C_{3} \leq C_{2}$.

Каждый объект и атрибут введены в граф только один раз. Атрибуты и объекты располагаются вдоль граней графа, как своего рода наследование. Атрибуты располагаются вдоль граней к вершине графа. Таким образом, высший элемент графа (верхние грани контекста) соответствует $\{\varnothing, M\}$. Элемент основания графа (нижние грани контекста) соответствует $\{G, \varnothing\}$. Для наглядности имена атрибутов отмечаются цифрами, а имена объектов отмечены буквами около узла графа.

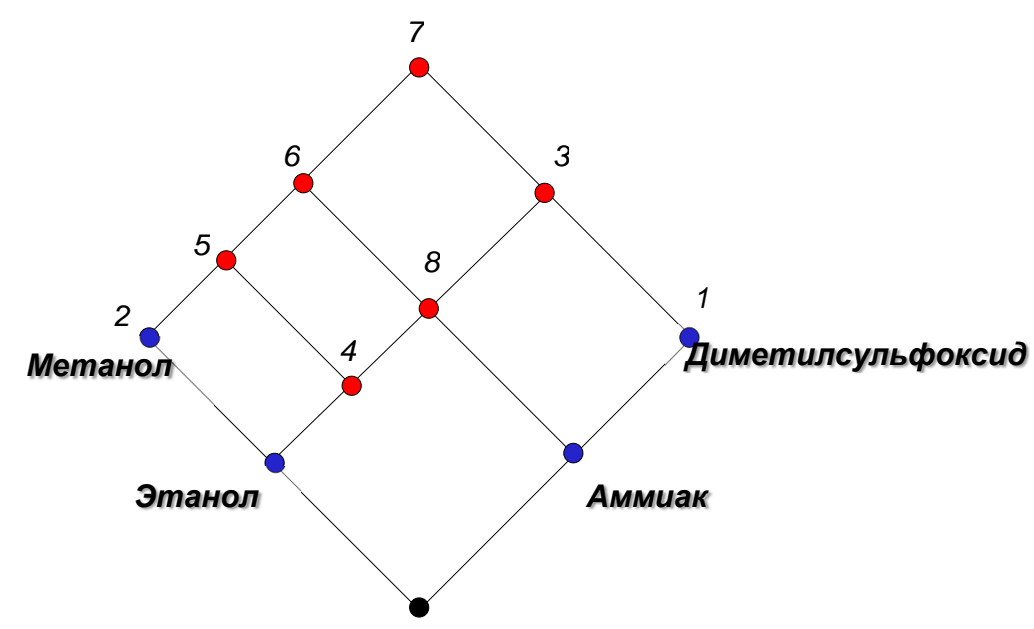

Рисунок 4 - Концептуальная решетка на примере четырех вредных веществ, для формирования логических правил идентификации вредных веществ

По решетке можно проследить все свойства, которыми обладает то или иное вещество: это множество всех свойств, лежащих выше узла, помеченного названием вредного вещества. Каждый узел решетки соответствует концепту.

Заметим, что если для всех объектов контекста, для которых справедливо некоторое свойство $\mathrm{P}$, справедливо также некоторое свойство $\mathrm{M}$, то импликация $P \rightarrow M$ является истинной. Иными словами, если импликация $P \rightarrow M$ истинна для контекста $\mathrm{K}=(\mathrm{Y}, \mathrm{X}, \mathrm{F})$ и к любому объекту $g \subseteq Y$ применим каждый признак из посылки $\mathrm{X}$, то к нему применим также признак из заключения импликации $\mathrm{Y}$, где $X \subseteq M_{\text {и }} Y \subseteq M$

В результате применения данного подхода разрабатываются правила логического вывода. Схема экспертно-информационной системы [20, 21] идентификации вредных веществ воздушной среде представлена на рисунке 5. Данная система включает 
следующие блоки: администратор диалога и решатель задач, который состоит из подсистем: процессора логического вывода, базы знаний, системы поддержки рассуждений (СПР).

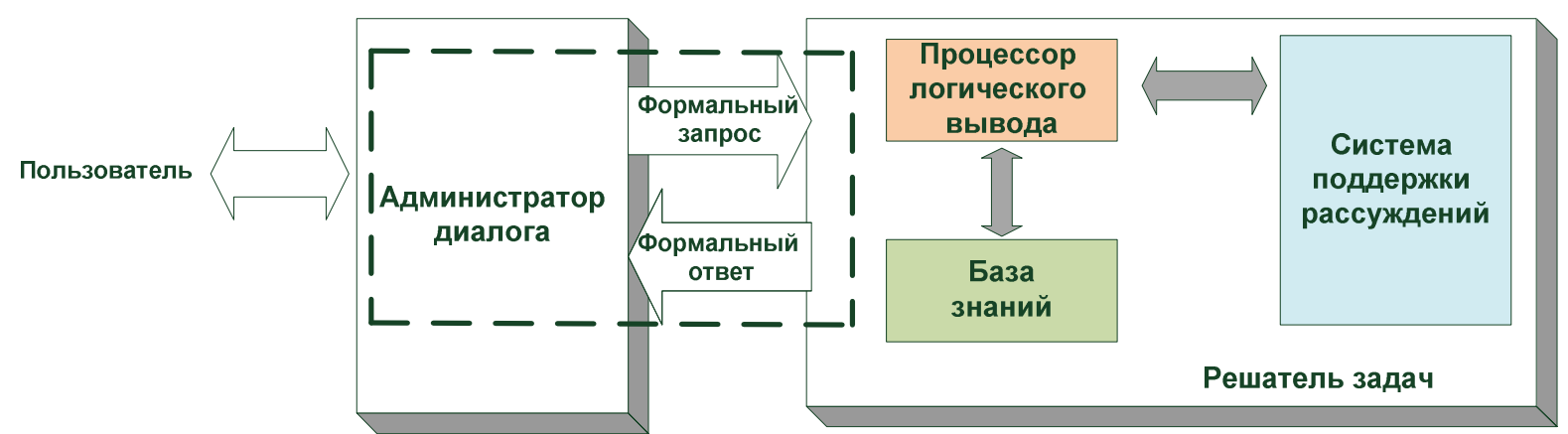

Рисунок 5 - Схема экспертно-информационной системы в составе информационно-вычислительного комплекса на основе логического формализма модифицированных рассуждений

Определение вредных веществ в воздушной среде представляет собой итерационный процесс. В ходе мониторинга формируется дополнительная информация в виде модифицируемых рассуждений. В связи с этим, процесс рассуждений определения вредных веществ в окружающей среде, является немонотонным [11-13].

Поэтому предлагается следующий общий алгоритм функционирования экспертно-информационной системы, построенной на немонотонном процессе вывода:

Шаг 1. Формирование фактов и правил в базе знаний (проводится экспертом).

Шаг 2. Из множества правил логического вывода находится правило, содержащее целевое утверждение (осуществляется решателем задач).

Шаг 3. Сопоставление найденного правила с имеющимися фактами (осуществляется решателем задач).

Шаг 4. Если образец правила при сопоставлении совпал с какими-либо фактами, то осуществляется выполнение этого правила (логический вывод) (осуществляется решателем задач).

Шаг 5. Сохранение осуществленного вывода (фактов и правил) в специальной области (система поддержки рассуждений).

Шаг 6. Если логический вывод противоречит некоторым фактам и заключениям, то выполняется сохранение в специальной области этих фактов и правил, а так же удаление их из базы знаний (система поддержки рассуждений).

Шаг 7. Если достигнут конец множества правил логического вывода, то переход к шагу 8, иначе переход к шагу 2.

Шаг 8. Завершение работы.

Для реализации немонотонности рассуждений разработана система поддержки рассуждений [6]. Процессор логического вывода информирует систему поддержки рассуждений, от каких данных зависит вывод, т.е. какие посылки и правила были использованы. Значимый, т.е. непротиворечивый вывод, передается в систему поддержки рассуждений, как обоснование. Вывод противоречия говорит о 
несовместимости предположений, в рамках которых сделан вывод. Таким образом, система поддержки рассуждений используется для защиты логической целостности и непротиворечивости заключений в экспертно-информационной системе, построенной на немонотонном логическом выводе. Взаимодействие между процессором логического вывода и СПР представлено на рисунке 6.

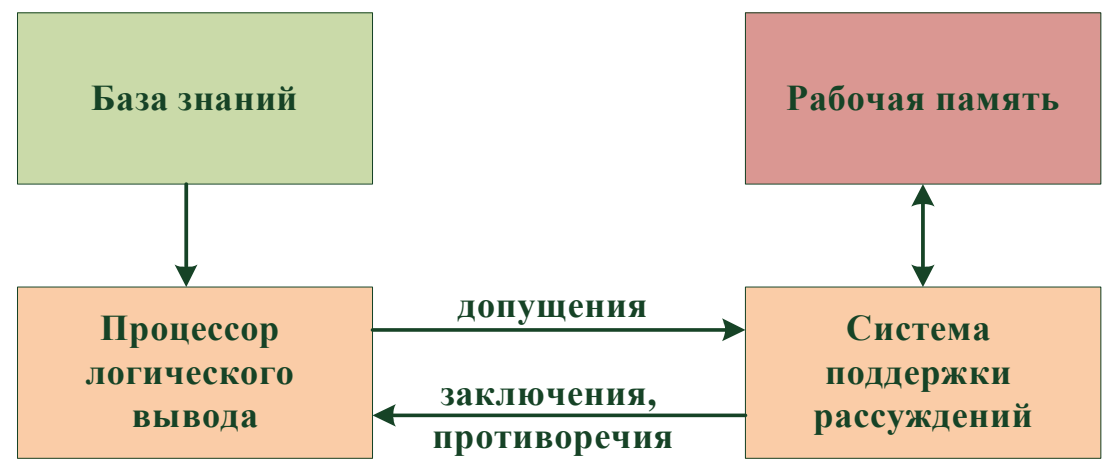

\section{Рисунок 6 - Взаимодействие процессора логического вывода и СПР}

Каждый факт в СПР представлен в виде узла. Зависимость заключения от фактов изображается в виде логического вентиля. Совокупность логических вентилей образует сеть обоснований [6].

\section{Определение концентрации вредных веществ}

Методы количественного анализа состава вредных веществ воздушного бассейна атмосферы основаны на построении зависимости между интенсивностью поглощения веществом ИК излучения и его концентрацией. На основе регистрации партии спектров ИК излучения вредных веществ с известными концентрациями устанавливается зависимость между спектром поглощения и концентрацией [7].

В данной работе для повышения скорости работы и точности определения концентрации вредного вещества из данных, полученных с систем лазерного зондирования и автоматизации построения функциональной зависимости между набором данных, полученных с систем лазерного зондирования, и концентрацией вещества предложено использовать метод нейронных сетей.

Использование нейронных сетей $(\mathrm{HC})$ в качестве аппарата для установления зависимости между интенсивностью поглощения веществом ИК излучения в окружающей среде и его концентрацией обусловлено способностями сети к параллельной и распределенной обработки информации, что позволяет значительно увеличить скорость работы алгоритма и повысить эффективность определения концентрации вредных веществ в воздушном бассейне атмосферы [7]. Кроме этого, нейросетевой метод моделирования не требует априорного задания вида исследуемой зависимости.

Задача определения концентрации вредного вещества по данным спектра поглощения может быть сформулирована как задача аппроксимации функции многих переменных. Необходимо построить некоторое отображение $\mathrm{H} \rightarrow \mathrm{Y}$ такое, чтобы на 
каждый возможный входной образ, представленный вектором спектра поглощения ИК излучения вредными веществом $(\mathrm{H})$ формировался правильный выходной вектор концентрации данного вещества (Y).

С помощью проведенного анализа нейронных сетей, с точки зрения решения задачи определения концентрации вредных веществ в воздушной среде, доказана возможность использования метода нейронных сетей для решения задачи автоматизации построения функциональной зависимости между спектром поглощения ИК излучения вредными веществами и их концентрацией [14-16].

Нейронная сеть может быть представлена как совокупность простых элементов (нейронов), связанных друг с другом. Нейрон состоит из нескольких входов и одного выхода, сумматора и блока функции активации [14]. Функционально нейрон умножает входные импульсы на некоторые коэффициенты (синаптические веса), суммирует полученные произведения и преобразует сумму в соответствии активационной функцией, являющейся пороговой или сигмоидальной. Математически функцию нейрона можно описать следующим образом:

$$
\boldsymbol{Y}=F\left(\sum_{i=1}^{n} w_{i} h_{i}+w_{0}\right)=F(\boldsymbol{W H})
$$

где W - весовые коэффициенты, Н - входные значения спектра, F - функция активации.

Для решения задачи установления концентрации вредных веществ по данных ИК спектра поглощения предложена многослойная нейронная сеть (рисунок 7):

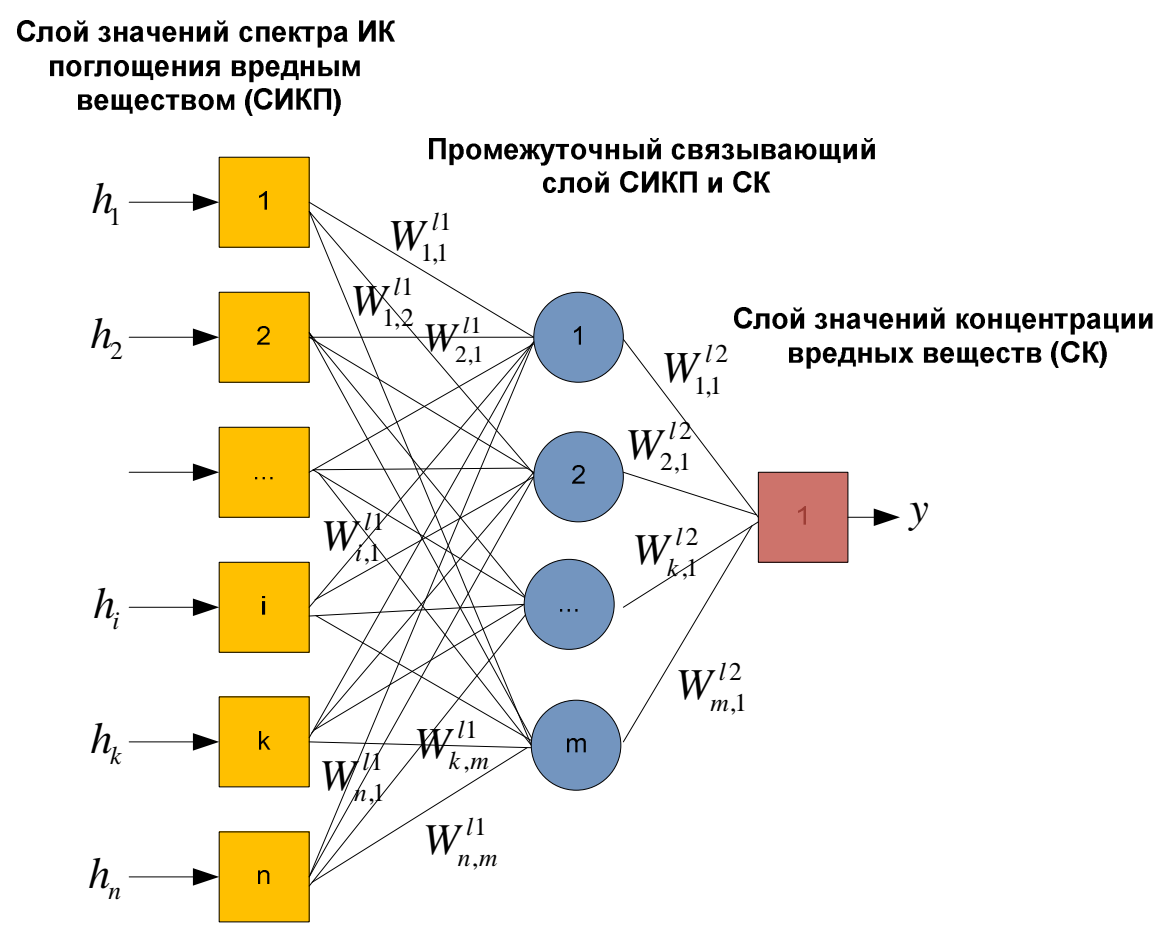

Рисунок 7 - Структура трехслойной нейронной сети, используемая для установления концентрации вредных веществ по данным ИК спектра поглощения 
1 слой состоит из значений $h_{1}, h_{2}, \ldots, h_{n}$ спектра поглощения ИК излучения вредными веществом;

2 слой является промежуточным слоем, для повышения точности установления функциональной зависимости между спектром поглощения и концентрацией вещества;

3 слой состоит из значения ${ }^{y}$ - концентрации вредного вещества, определяемой из спектра поглощения, $y \in \boldsymbol{Y}$.

Каждый слой связан следующими весами:

$W_{i, j}^{l 1}$ — возбуждающий вес между i-м нейроном СИКП слоем и ј-м нейроном промежуточного слоя;

$W_{k, 1}^{l 2}$ - возбуждающий вес между i-м нейроном промежуточного слоя и j-м нейроном слоя СК.

Обучение нейронной сети основано на обучении с учителем, заключающееся в том, что каждому входному набору данных $h_{1}, h_{2}, \ldots, h_{n}-$ значений спектра поглощения ИК излучения регистрируемого вещества ставится в соответствие выходное значение ${ }^{y}$ - концентрация вредного вещества.

Обучение с учителем определено как поиск коэффициентов $W_{i, j}^{l 1}{ }_{\text {и }}^{l 2} W_{k, 1}^{l}$ нейронной сети, при которых будет выполняться необходимое отображение входного вектора $\boldsymbol{H}$ значений спектра поглощения в выходное Ү - значение концентрации. Это достигается путем минимизации суммы разности квадратов между желаемыми выходами и получаемыми нейронной сетью [16]:

$$
E=\frac{1}{2} \sum_{i}^{M} \sum_{j}^{N}\left(y_{j}\left(W, H_{i}\right)-d_{i, j}\right)^{2} \rightarrow \min ,
$$

где $d_{i, j}$ - желаемый выход j-го выходного нейрона для i-го обучающего примера; $W$ - матрица весовых коэффициентов нейронной сети, коэффициенты одного нейрона представлены строкой матрицы $H_{i}-$ i-ый обучающий пример.

С целью выбора алгоритма обучения нейронной сети исследованы различные алгоритмы: эволюционные, стохастические, градиентные [16]. В качестве метода обучения использовался модифицированный метод обратного распространения ошибки [14]: На основе анализа различных параметров сети (вид нейронов, количество слоев и связей, типы сетей), выполненного с целью получения оптимальной нейронной сети для решения задачи определения концентрации вредных веществ по данным поглощения вредным веществом ИК излучения, предлагается пошаговый алгоритм редукции сети с учетом конкуренции связей нейронов между собой:

Шаг 1. Задание исходных значений для каждого элемента (нейрона). 
Шаг 2. Ввод значений возбуждающих весовых коэффициентов $W_{i, j}^{l 1}$ - степень связи, с которой і-ый нейрон связан с ј-ым нейроном следующего слоя.

Шаг 3. Вычисление значений каждого нейрона по формуле:

$$
\begin{aligned}
g_{i} & =\frac{S_{i}}{K}, \\
S_{i} & =\sum_{j=1}^{m} W_{i, j}^{l 1} x_{j}
\end{aligned}
$$

где $S_{i}$ - сумма произведений всех возбуждающих весов і-ого нейрона на значение соответствующего этому весу ј-ого нейрона следующего слоя, gj - величина ј-ого элемента следующего слоя, $\mathrm{K}=1$ - коэффициент, который используется для обновления значений нейронов предыдущего слоя, $\mathrm{i}$ - принимает значение от 0 до n, n количество нейронов предыдущего слоя, $\mathrm{j}$ - принимает значение от 0 до $\mathrm{m}, \mathrm{m}-$ количество элементов следующего слоя нейронной сети.

Шаг 4. Обновление значений возбуждающих весов.

$$
W_{i, j}^{l 1}=\frac{W_{i, j}^{\prime l 1} \cdot \sum_{i}\left(W_{i, j}^{\prime 1}\right)}{\left(1-x_{i} x_{j}\right)},
$$

где $\stackrel{l 1}{\prime}_{i, j}$ - значения возбуждающих весов предыдущей итерации.

Шаг 5. Если $\stackrel{l 1}{W_{i, j}} \leq 0,001$, то $\stackrel{l 1}{W_{i, j}=0}$. Таким образом происходит сокращение количества конкурируемых между собой нейронов.

Шаг 6. Вычисление обновленных значений по формуле (2).

Шаг 7. Исследование значений нейронов на противоречие в конкуренции:

$$
\begin{aligned}
& I_{i}=\sum_{k=1}^{n} W_{i, k}^{p r} \cdot x_{i}, k \neq i, I_{i} \geq 0
\end{aligned}
$$

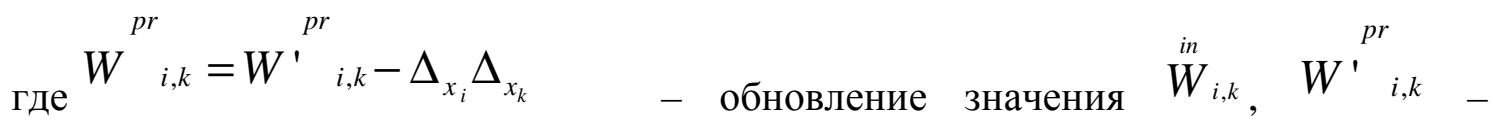
${ }_{\text {значение }} W^{p r} i, k$ на предыдущей итерации, $\Delta x_{i} \Delta x_{k}$ - разница значений хі и хk на двух итерациях.

Шаг 8. Вычисление новых значения нейронов с учетом учета противоречия в конкуренции:

$$
x_{i}=\frac{S_{i}}{K+I_{i}}
$$


Шаг 9. Если значения нейронов не превышают установленного порога, то изменение весов у нейронов прекращают. Иначе продолжается изменение весов и пересчет значений (переход к шагу 4).

Алгоритм является итерационным. Он используется для установления наиболее конкурентных связей в нейронной сети.

Предложенный метод определения количественного состава вредных веществ в воздушном бассейне атмосферы по данным ИК спектрометрии, основанный на нейросетевых технологиях, позволяет повысить точность в установлении концентрации на $45 \%$, повысить скорость обработки информации на $25 \%$, и в целом увеличить эффективность функционирования систем экологического мониторинга, использующих нейросетевые технологии в установлении количественного состава, на $30 \%$.

\section{Разработка информационно-технического комплекса}

В рамках предложенной концепции проведена апробация разработанных моделей и алгоритмов интеллектуального мониторинга воздушного бассейна окружающей среды с использованием лазерного ИК зондирования результатом которой стала разработка соответствующего информационно-технического комплекса.

Информационно-вычислительный комплекс предназначен для автоматизации принятия решений о наличии вредных веществ в воздушной среде и их концентрации. Информационно-технический комплекс позволяет автоматизировать выполнение следующих задач: представление данных; идентификация вредных веществ в окружающей среде с использованием логического формализма модифицированных рассуждений; определение концентрации вредных веществ с помощью искусственных нейронных сетей; обучение и настройка нейронной сети; визуализация результатов. Разработана система различных меню, многопользовательский интерфейс, набор управляющих элементов. Комплекс работает под управлением операционных систем семейства Windows.

Исследована эффективность разработанного информационно-вычислительного комплекса. Осуществлена проверка его работоспособности на предприятиях, занимающихся мониторингом окружающей среды. Определены основные техникоэкономические показатели работы (таблица 2). 
Таблица 2 - Сравнение технико-экономических показателей предлагаемого решения

\begin{tabular}{|c|c|c|c|c|}
\hline Показатель & $\begin{array}{c}\text { Опытный } \\
\text { образец }\end{array}$ & $\begin{array}{c}\text { АС } \\
\text { дистанционного } \\
\text { мониторинга } \\
\text { «АСД Лидар» } \\
\text { (Россия) }\end{array}$ & $\begin{array}{c}\text { УПГ-ЛИМБ } \\
\text { (ЗАО } \\
\text { «Испытательно- } \\
\text { метрологичес-кая } \\
\text { лабора-тория } \\
\text { России) } \\
\end{array}$ & $\begin{array}{c}\text { Sabre } \\
2000 \\
\text { (Smiths } \\
\text { Detection, } \\
\text { Great } \\
\text { Britain) } \\
\end{array}$ \\
\hline $\begin{array}{lr}\text { Использование } & \text { труда } \\
\text { экспертов } & \text { при } \\
\text { идентификации } & \\
\text { вредных веществ } & \\
\end{array}$ & нет & да & да & да \\
\hline $\begin{array}{l}\text { Количество } \\
\text { идентифицируемых } \\
\text { веществ в атмосфере } \\
\text { при одном измерении }\end{array}$ & 8 & 1 & 1 & 1 \\
\hline Быстродействие (сек) & 10 & 15 & 12 & 15 \\
\hline $\begin{array}{l}\text { Возможность } \\
\text { установления } \\
\text { концентрации } \\
\text { вредных веществ }\end{array}$ & да & нет & нет & нет \\
\hline $\begin{array}{l}\text { Стоимость расходных } \\
\text { материалов для } \\
\text { обслуживания } \\
\text { прибора (руб. в год) }\end{array}$ & 5000 & 500000 & 10000 & 300000 \\
\hline Радиус действия (км) & 25 & 25 & На месте (0) & $\begin{array}{c}\text { На месте } \\
(0)\end{array}$ \\
\hline Цена (тыс. руб) & $\begin{array}{c}100,00 \\
\text { (при серийном } \\
\text { производстве) } \\
\end{array}$ & 1845,00 & 365,80 & 500,00 \\
\hline
\end{tabular}

Ошибки определения состава вредных веществ в воздушной среде показаны на рисунке 9.

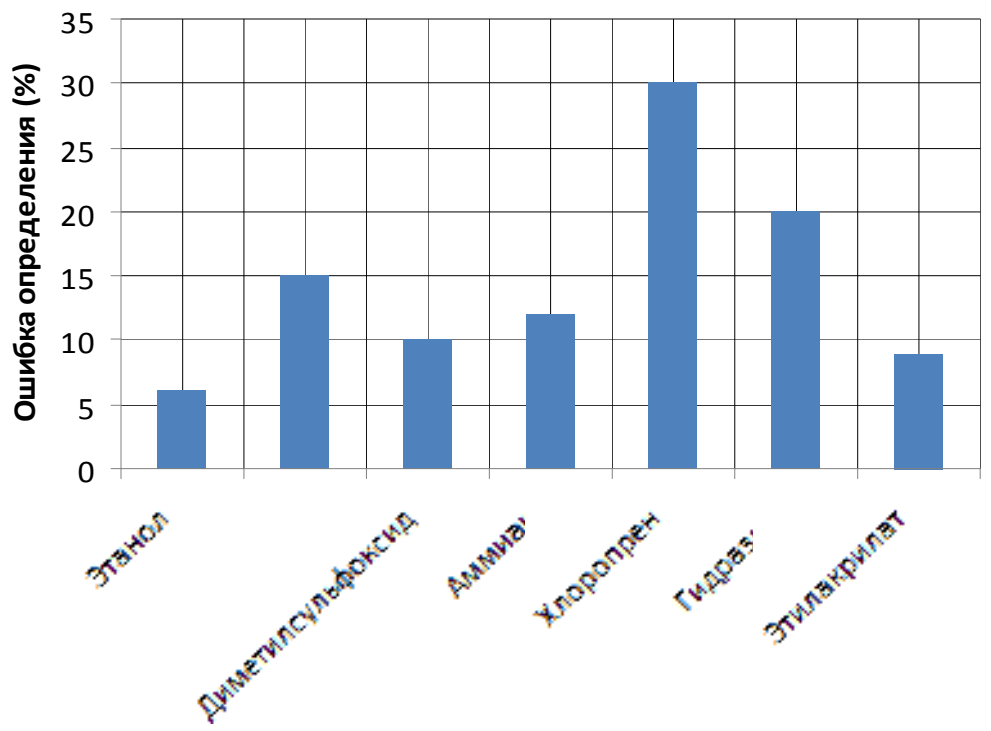

Рисунок 9 - Ошибки определения вредных веществ в воздушной среде 
Зависимость вероятности идентификации вредных веществ от накопленных в информационно-техническом комплексе правил логического вывода представлена на рисунке 10.

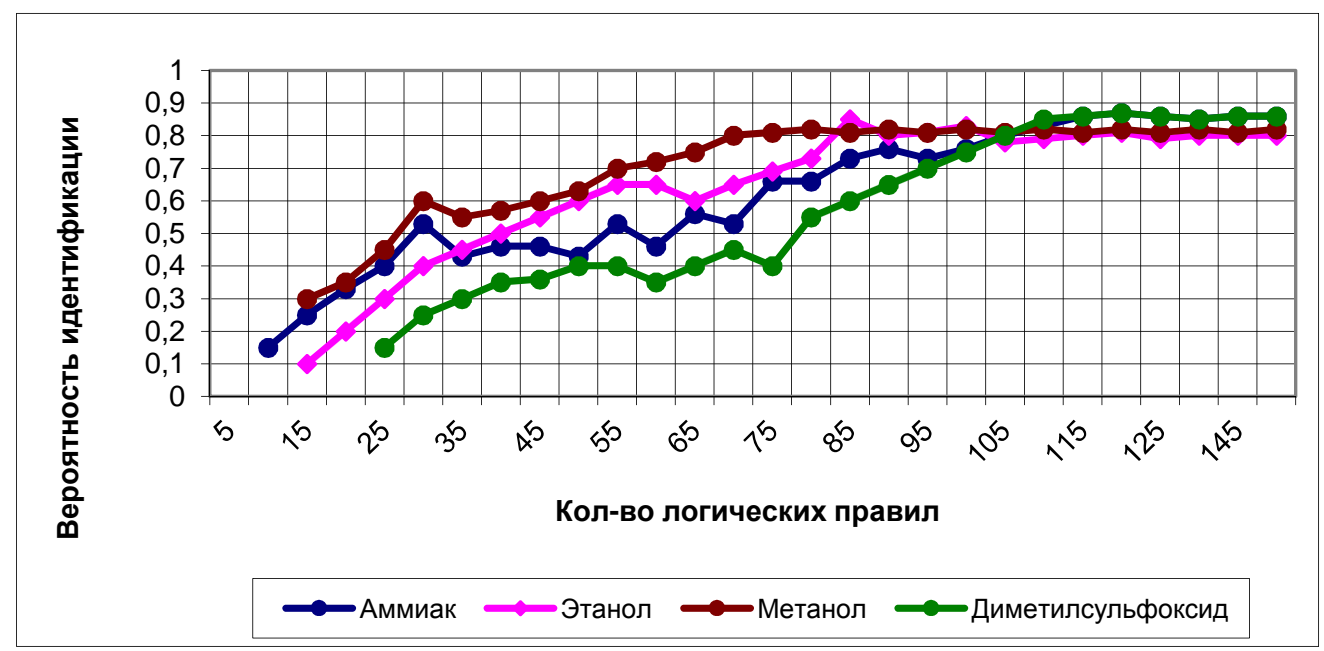

Рисунок 10 - Зависимость вероятности идентификации вредных веществ от количества накопленных в информационно-вычислительном комплексе логических правил

\section{Заключение}

Таким образом, был проведен анализ современных моделей, методов и алгоритмов обработки и автоматизированной идентификации сигналов для систем экологического контроля (мониторинга) окружающей среды с использованием лазерного ИК зондирования. Разработана концепция интеллектуального дистанционного мониторинга и контроля объектов специального назначения. Предложена модель принятия решений о наличии вредных веществ в воздухе атмосферы и разработан алгоритм реализации данной модели на основе использования логического формализма модифицируемых рассуждений. Разработан метод определения концентрации вредных веществ в атмосфере на основе нейросетевых технологий с помощью специального представления спектра поглощения ИК излучения, полученных с лазерных систем дистанционного зондирования воздушного бассейна атмосферы.

Предлагаемая концепция и структура информационно-технического комплекса может быть использована для определения наличия вредных веществ и их концентрации в воздушной среде с использованием данных, полученных от систем лазерного ИК зондирования; в системах экологического мониторинга и контроля объектов специального назначения; для мониторинга наличия вредных веществ автомобильных трасс г. Москвы. Информационно-технический комплекс возможно использовать для работ в ООО КБ «ЭлектронСистема», а также в следующих структурах: в службах безопасности и охраны труда, экологических и санитарных лабораториях, промышленных предприятия, инспекционных службах 
Госкомприроды, Санэпидемнадзора и МЧС, промышленных предприятиях, предприятиях энергетики, научных, исследовательских и фармацевтических предприятиях, транспортных компаниях, складских помещениях.

Кроме этого потенциальными пользователями и заказчиками проекта могут являться следующие структуры государственного управления и организации: службы МВД и ФСБ, в том числе: Научно-производственный центр антитеррористической и криминалистической техники Спектр-АТ; федеральные и городские службы безопасности и охраны труда; городские лаборатории экологического и санитарного надзора; организации промышленности г. Москвы и Московской обл., осуществляющие обрабатывающее производство: ФГУП ММПП «Салют», ОАО «Лыткаринский завод оптического стекла», ОАО «Московский завод «САПФИР», ОАО «ПЬЕЗО», ФГУП НПО «Орион», ОАО Концерн «Моринформсистема-АГАТ» и другие; предприятия энергетики; транспортные компании: аэропорты, вокзалы, метрополитен; предприятия, оказывающие складские услуги.

\section{Список информационных источников}

[1] Морозова Т.Ю. Основные тенденции развития автоматизации передовых отраслей промышленности. // Промышленные АСУ и контроллеры. 2010. № 12. С. 12-14.

[2] Козинцев В. И., Орлов В. М., Белков М. Л. Оптико-электронные системы экологического мониторинга природной среды. М.: Изд-во МГТУ им. Н. Э. Баумана, 2002. - 527c.

[3] Агишев Р.Р. Лидарный мониторинг атмосферы. - М.: ФИЗМАТЛИТ, 2009. $316 \mathrm{c.}$

[4] В.Е. Зуев, В.В. Зуев Дистанционное оптическое зондирование атмосферы. СПб.: Гидрометеоиздат, 1992. - 232с.

[5] Никонов В.В. Принципы построения промышленных автоматизированных систем обработки сигналов лидарных систем для дистанционного зондирования атмосферы. // Промышленные АСУ и контроллеры, 2010. № 3, с. 7-8.

[6] Никонов В.В. Построение модели экспертно-информационной системы на основе логического формализма в задачах экологического мониторинга // Приборы и системы. Управление. Контроль. Диагностика, 2010. № 4, с. 9-14.

[7] Никонов В.В. Использование нейросетевых технологий для определения количественного состава вредных веществ в воздушном бассейне атмосферы по данным ИК спектрометрии // Экологические системы и приборы № 5, 2010. - с. $16-19$.

[8] Биркгоф Г. Теория решеток.-М.: Наука, 1984, 337 с.

[9] Ganter B., Wille R. Formale concept analysis: mathematical foundatioun.-Springer Verlag, New York, 1997, 93 c.

[10] Клюха А.А., Морозова Т.Ю. Об одном методе анализа данных в задаче психологической диагностики. // Информационно-управляющие системы. 2007. № 5. C. 42-44.

[11] Ашинянц Р.А. Логические методы в искусственном интеллекте. М.: МГАПИ, 1996.

[12] Doyle J. Truth Maintenance Systems // J. of Artificial Intelligence, vol. 12, 1979

[13] Вагин В.Н., Головина Е.Ю, Загорянская А.А. Достоверный и правдоподобный вывод в интеллектуальных системах. - М.: ФИЗМАТЛИТ, 2004. - 704 с. 
[14] Круглов В.В., Борисов В.В. Искусственные нейронные сети. Теория и практика. - М.: Горячая линия-Телеком, 2001.

[15] Морозова Т.Ю., Бурлаченко Т.Б. Нейросетевая оптимизация абдуктивных выводов в задачах диагностики технических систем. // Мехатроника, автоматизация, управление. 2008. № 8. С. 19-23.

[16] Осовский С. Нейронные сети для обработки информации. / Пер. с польского И.Д. Рудинского. - М.: Финансы и статистика, 2004.

[17] Клюха А.А., Никонов В.В. Использование логико-алгебраического подхода для формирования логических правил идентификации вредных веществ в воздушном бассейне атмосферы // Промышленные АСУ и контроллеры. 2010. № 5. С. 24-26.

[18] Ветлугин М.М. Автоматизация мониторинга состояния среды промышленных предприятий / М.М. Ветлугин, К.С. Колдашев, А.Л. Рябикин, А.В. Остроух // Приборы и системы. Управление, контроль, диагностика. - М.: «Научтехлитиздат», 2007. - №2. - С. 13-16.

[19] Бакатин Ю.П. Инновации в экологическом контроле строительных и дорожных машин / Ю.П. Бакатин, А.В. Остроух // Наука и техника в дорожной отрасли. М.: ЗАО «Издательство дороги», 2011. - №3. - С. 35-38.

[20] Остроух А.В. Основы построения систем искусственного интеллекта для промышленных и строительных предприятий: монография / А.В. Остроух. - М.: ООО «Техполиграфцентр», 2008. - 280 с. - ISBN 978-5-94385-033-2.

[21] Остроух А.В. Системы искусственного интеллекта в промышленности, робототехнике и транспортном комплексе: монография / А.В. Остроух Красноярск: Научно-инновационный центр, 2013. - 326 с. - ISBN 978-5-90631410-9. 